\title{
The SCore Cluster Enabled OpenMP Environment: Performance Prospects for Computational Science
}

\author{
H'sien. J. Wong and Alistair P. Rendell \\ Department of Computer Science, Australian National University, \\ Canberra ACT0200, Australia \\ alistair.rendelleanu.edu.au
}

\begin{abstract}
The OpenMP shared memory programming paradigm has been widely embraced by the computational science community, as has distributed memory clusters. What are the prospects for running OpenMP applications on clusters? This paper gives an overview of the SCore cluster enabled OpenMP environment, provides performance data for some of the fundamental underlying operations, and reports overall performance for a model computational science application (the finite difference solution of the 2D Laplace equation).
\end{abstract}

\section{Introduction}

The two main classes of parallel computers available in today's markets are clusters and hardware enabled Shared Memory Systems (SMS). Clusters are assembled from multiple disjoint computers and are generally programmed using some form of message passing. SMS on the other hand have a single common address space and can be programmed using either message passing or various threaded programming models. In general SMS are considered easier to use, but due to the need for specialized hardware they are also more expensive, and this is especially true for high processor counts.

OpenMP [1] is a threaded programming model widely used on SMSs. Essentially it provides a compiler based interface to an underlying thread library. The model is attractive since it permits the incremental parallelization of existing sequential application codes and, as it consists largely of compiler directives, a developer can easily support both parallel and sequential versions of the same code at the same time.

Given the cost advantages of clusters, but the programming advantages of OpenMP and the existence of a large body of OpenMP code, it is not surprising that various groups have been attempting to develop OpenMP programming environments for clusters [2-5]. Most of these attempts have been based on layering OpenMP on top of some existing Software Distributed Shared Memory (SDSM) environment, although we note interesting recent work by Huang et al to implement OpenMP over Global Arrays [6]. In either case, to obtain reasonable performance from an OpenMP code on a cluster it is likely that the application programmer will require some knowledge of the implementation. The aim of this paper is to discuss these issues in relation to the SCore cluster enabled OpenMP environment $[2,7]$ and to analyze its performance on 
a cluster comprising dual $550 \mathrm{MHz}$ Pentium III processors linked via a 100MBit Ethernet interconnect.

\section{The SCore Distributed Shared Memory Environment}

The SCore cluster enabled OpenMP is layered on top a page based SDSM environment called SCASH [2]. A basic understanding of SCASH is critical to understanding the overall performance of the SCore cluster enabled OpenMP. Essentially SCASH separates the address space of each process into global and local memory pages. Data assigned to local memory pages is private to each process, while data in the global memory pages can be shared between all processes. To facilitate sharing of the global memory pages, read and write accesses to the corresponding address ranges are protected using mprotect (on Unix). This means that when any process first accesses data in a global memory page an interrupt is triggered and this induces execution of the relevant SCASH interrupt handler. The interrupt handler determines the location of the required page and makes it available to the requesting process. There are three possible home locations for the requested page; i) it has been assigned to memory associated with the calling process; ii) it has been assigned to memory associated with another process that resides on the same physical computer as the calling process; iii) it has been assigned to memory associated with a process that resides on another physical computer. In case $\mathrm{i}$ the requested memory page is available immediately; in case ii the page might be available in, e.g. a shared memory segment that is linked to both processes; while in case iii a transfer of the page over the communication network is required.

The interrupt is further characterized by the type of interrupt. Global memory pages assigned to the calling process or to a process located on the same node as the calling process are initially given read access, thus interrupts involving these "home" or "local" pages only occur when writing to these pages. All "remote" global memory pages are initially marked as "unmapped", so depending on the operation transitions unmapped-read, unmapped-write or read-write can occur. Indeed if a process first reads from one of these pages and then shortly afterwards writes to the same page two interrupts will occur, one causes an unmapped-read transition and the other causes a read-write transition. In this case it would obviously be better to have a single interrupt with unmapped-write transition, and while in some cases the compiler may be able to make such optimizations this should not be taken for granted.

To permit multiple processes to simultaneously access global data, copies of the same memory page can be transferred to multiple different processes. If the requesting process only requires read access this does not present a problem. If, however, it requires write access then there are two considerations. First, when do the modifications become visible to all other processes. Second and since memory pages are typically large (e.g. 4096bytes or greater), how to support multiple simultaneous writes to disjoint regions of the same memory page.

The first issue, also known as the memory consistency model, is enforced by SCASH at synchronization points. This means that modifications made to any of the global memory pages are propagated back to the page owners at every synchronization point. Moreover if one process has modified a page, but another 
process has a read only copy of that page, then the read only copy must be invalidated and the protection on that page reset. This requires inter-process communication to communicate the changes, and some book keeping to keep track of which processes have copies of which pages. Whether this requires communication over the cluster interconnect will depend on the exact location of the process modifying a page compared to the owner of the page.

To handle multiple simultaneous updates to the same memory page, SCASH employs a "twinning and diffing" procedure. This means that if a write fault is encountered, as well as locating and fetching a copy of that page (if it is not already available to that process either because it is owned by that process or has been fetched via a read fault) the handler will create two copies. One is modified in subsequent write operations, while the other is left unmodified. At the next synchronization point, a "diff" is made between the modified and unmodified copy and the changes relayed to the process owning that page. The time required to communicate the differences will depend in part on the number of changes made to that page.

Table 1. The different memory page faults encountered in SCASH, details of what communications are required, and approximate times as recorded on a cluster of dual $550 \mathrm{MHz}$ Pentium III processor nodes linked via 100MBit/sec Ethernet. See text for further details

\begin{tabular}{cccccccc}
\hline $\begin{array}{c}\text { Access } \\
\text { Type }\end{array}$ & $\begin{array}{c}\text { Current } \\
\text { Permission }\end{array}$ & $\begin{array}{c}\text { Page } \\
\text { Owner }\end{array}$ & Abbreviation & $\begin{array}{c}\text { Send } \\
\text { Request }\end{array}$ & $\begin{array}{c}\text { Fetch } \\
\text { Page }\end{array}$ & $\begin{array}{c}\text { Create } \\
\text { Twin }\end{array}$ & $\begin{array}{c}\text { Time } \\
\text { (usec) }\end{array}$ \\
\hline Write & ReadOnly & Home & WROH & & & Yes & 8 \\
Write & ReadOnly & Local & WROL & Yes & & Yes & 51 \\
Write & ReadOnly & Remote & WROR & Yes & & & 7 \\
& & & WrWH & & & & 16 \\
Write & ReadWrite & Home & WRWH & & & 24 \\
Write & ReadWrite & Local & WRWL & Yes & & & 599 \\
Write & ReadWrite & Remote & WRWR & Yes & & & 25 \\
& UnMapped & Remote & WUMR & Yes & Yes & Yes & 597 \\
Read & ReadWrite & Remote & WRWR & Yes & & & 18 \\
Read & ReadOnly & Remote & RROR & Yes & & & 587 \\
Read & UnMapped & Remote & RUMR & Yes & Yes & & \\
\hline
\end{tabular}

Table 1 summaries the above and reports timing data for a variety of different page fault transitions obtained by running a set of specially designed OpenMP benchmarks under SCore version 5.4.0 on the Pentium III cluster. "Access type" denotes whether the interrupt was caused by a read or a write fault, "current permission" reflects the page permissions prior to the memory fault, and "page owner" denotes which process is ultimately responsible for this memory page. In this respect "home" implies that the process posting the interrupt is also the owner of the memory page, while "local" implies that the page is owned by a process running on the same physical node (noting the use of dual CPU nodes). Within SCASH not all transitions are possible, for example an RUMH transition is impossible since the default permission for a home page is ReadOnly. 
By way of contrast the timing data given in Table 1 should be compared to the latency of a "normal" memory access. This was measured using LMbench [8] as roughly 0.14 usec. Within SCASH this would be the cost of accessing local memory, the cost of making a read access to a global memory page owned by the calling process, or the cost of reading (writing) to a page of global memory once a local copy had been created and assigned read (readwrite) protection. Clearly, from the data given in Table 1 the cost of the first write to any page of global memory, or first read of a remote page is significantly more expensive than 0.14 usec. For example the cost of the first write to a global memory page owned by the calling process (WROH) is roughly 50 times greater at 8 usec. While writing to a global memory page located within the same node, but not owned by the calling process (WROL) is longer still, since it requires some book-keeping (denoted by "send request") and creation of a twin. Not surprisingly the most costly page faults involve read or write requests to an unmapped global memory page (WUMR or RUMR) as it requires that page to be transferred across the interconnect; at around 600usecs on a machine with a clock cycle of $\approx 2$ nsec this corresponds to roughly half a million clock cycles!

For the application programmer it is important to realize that the costs given in Table 1 will, in general, be encountered for the first access to global memory after every synchronization point. That is following a synchronization point any local copy of a memory page is likely to be invalidated, so subsequent reads or writes to that page will encounter a new page fault and cost penalty as detailed in Table 1.

A clear implication from the timing data is that codes which access memory by jumping from page to page with little or no reuse of data in the same page will perform very poorly (e.g. pointer chasing). Conversely to obtain reasonable performance the costs given in Table 1 need to be amortized over many subsequent data accesses to the same page, and this is especially true for remote page accesses. In short if you drag a memory page over the network you'd better make good use of it!

Finally, we note that some of the events given in Table 1 may appear a little strange. For instance, a WRWH page fault is encountered when two processes share the same computer, the owner of the page has not yet written to it, but a companion thread on the same node has. Thus when the owner thread accesses this page an interrupt is triggered, but the page has actually already been marked with readwrite access.

\section{SCore Cluster Enabled OpenMP}

With a basic understanding of SCASH we can now consider the performance of some key OpenMP synchronization directives. To do this we have used the OpenMP microbenchmark tests suite [9] developed at Edinburgh Parallel Computing Centre (EPCC). Before presenting the results, however, it is pertinent to outline briefly how OpenMP is mapped onto the underlying SCASH SDSM.

Not surprisingly OpenMP data quantities that are declared to be shared are stored in global SCASH memory pages. Thus if every thread in an OpenMP parallel region accesses the same global variable this will induce an interrupt on virtually all threads with requests for the relevant page to be transferred to the calling thread. The only exceptions are for read accesses to data stored in memory pages that are either owned 
by the calling thread or by a thread co-located on the same node. Thus as implemented the cost of transferring shared data from the master to child threads scales as $O\left(N o \_T h r e a d s\right)$. Of course if multiple shared data quantities are stored in the same page then accesses to these other quantities will be cheap once the initial page transfer has occurred.

The cluster enabled OpenMP compiler also uses a portion of the SCASH global shared memory space for administrative purposes. For example if the "\#pragma omp parallel" directive is combined with a "copyin" clause then threadprivate data is transferred between the master and child threads by placing the relevant data items into a global memory page and having each child thread retrieve this data as required. Similarly information relating to the scheduling of parallel loops and reduction operations are communicated through global memory pages. Also and as is common to most OpenMP implementations, threads once created are kept alive but dormant between parallel regions. The parallel regions are "outlined" as functions that are then called as applicable by the child threads on entry to a parallel region. To communicate the name of the relevant function between the master and child threads the name of the outlined function is placed in an global memory page.

Synchronization operations in OpenMP map to synchronization operations in SCASH. As part of the SCASH consistency model discussed above, this is where memory pages are flushed and updated. An SCASH barrier consists of 5 phases.

1. Synchronization

2. Flushing of modified memory pages

3. Synchronization

4. Invalidation of pages

5. Synchronization

Here flushing of the modified memory pages involves transferring the differences that have resulted from write operations back to the owning page, while invalidation of pages involves communicating with remote processes so that they can update their page tables based upon which pages have been modified. Just from this basic understanding of what is involved it is clear that synchronizations will be expensive.

In Table 2 we report the timing results for the EPPC OpenMP synchronization microbenchmarks run on the Pentium III cluster. Some minor modifications to the test suite were made. In particular the iteration counter used in these benchmarks is assigned global scope. Since the first access to this variable (or more precisely the global memory page containing this variable) occurs within the timing routine this induces an interrupt and transfer of the associated memory page to the requesting page. To avoid this additional overhead we have defined this variable as threadprivate with a copyin clause to transfer it from master to child before the start of the timed loop.

The results obtained on the Pentium cluster and using between 1 and 8 OpenMP threads are compared with similar data obtained on a Sun V1280 system with 12 $900 \mathrm{MHz}$ UltraSparc III processors and hardware shared memory. On the cluster results were obtained using both 1 and 2 threads per dual processor Pentium III node. Comparing a single thread run on the cluster (denoted $1 \mathrm{x} 1$ ) with a single thread run on the Sun (denoted 1) we see that the overheads associated with inclusion of the OpenMP directives are roughly equivalent (especially when the faster clock rate of 
the Sun processor is considered). As soon as we move to multiple threads, however, the situation changes dramatically with significantly larger overheads recorded on the cluster. Moreover this is even true when running 2 OpenMP threads on 1 node of the cluster (denoted 1x2) where the cost of the parallel/for/barrier/single/ reduction constructs are typically two orders of magnitude larger than the equivalent results obtained on the Sun. If we move to 2 threads running across 2 nodes of the cluster (denoted 2x1) the performance of these operations gets even worse. In comparison the overhead associated with the critical/ordered/ atomic directives is relatively good for two threads within the same nodes, but increases dramatically when the threads are located on different nodes.

Table 2. Overhead (usec) for OpenMP synchronization directives on cluster with dual processor Pentium III nodes linked via 100MBit/sec Ethernet and a $12900 \mathrm{MHz}$ CPU Sun hardware shared memory V1280 system

\begin{tabular}{lccccccccc}
\hline & \multicolumn{1}{c}{ Pentium III (nodes x threads/node) } & \multicolumn{3}{c}{ Sun V1280 (Threads) } \\
Directive & $\mathbf{1 x 1}$ & $\mathbf{2 \times 1}$ & $\mathbf{1 x 2}$ & $\mathbf{4 x 1}$ & $\mathbf{4 x 2}$ & $\mathbf{1}$ & $\mathbf{2}$ & $\mathbf{4}$ & $\mathbf{8}$ \\
\hline parallel & 0.8 & 1762 & 474 & 13556 & 43571 & 0.3 & 5.3 & 6.8 & 10.0 \\
for & 0.5 & 662 & 221 & 7731 & 17033 & 0.5 & 2.1 & 2.9 & 4.3 \\
parallel for & 1.2 & 1797 & 471 & 13498 & 42571 & 0.7 & 6.0 & 7.3 & 12.8 \\
barrier & 0.2 & 661 & 225 & 7305 & 17631 & 0.1 & 1.1 & 1.8 & 2.9 \\
single & 0.9 & 4371 & 304 & 15330 & 47804 & 0.1 & 0.9 & 1.3 & 2.0 \\
critical & 1.3 & 179 & 6 & 260 & 976 & 0.2 & 0.3 & 0.5 & 0.5 \\
lock & 0.6 & 52 & 4 & 74 & 204 & 0.2 & 0.4 & 0.5 & 0.5 \\
ordered & 1.7 & 1154 & 8 & 3712 & 6454 & 0.2 & 0.6 & 0.6 & 0.6 \\
atomic & 1.3 & 3446 & 7 & 4776 & 5023 & 0.1 & 0.5 & 0.8 & 1.1 \\
reduction & 1.1 & 29994 & 966 & 47836 & 98553 & 0.5 & 5.5 & 7.8 & 12.0 \\
\hline
\end{tabular}

Table 2 shows that on the cluster the cost of a parallel/parallel for directive is roughly twice the cost of a barrier, while the cost of an isolated for directive is roughly equal to the cost of a barrier. This is easily explained by the existence of an implicit barrier at both the start and end of the parallel and parallel for directives, but only one implicit barrier at the end of the for directive. In contrast the single directive, while also containing a barrier at the end of the associated region of code, also requires some additional book-keeping to ensure that just one thread executes this portion of code. This is handled by using a shared counter, with access to this counter controlled by locks. As the shared counter is stored in global memory, page faults are encountered when each thread accesses it giving rise to extra cost. Also the overall cost of the single directive appears to scale rather poorly with increasing thread count, thus depending on the context, it may be better to specifically assign the work/code associated with this directive to one thread.

The most expensive operation is reduction. This requires two barrier calls, and also makes use of the global administrative pages. Specifically, prior to the first barrier call, all threads place their partial results into unique locations in the global 
administrative page. The first barrier serves to propagate these partial contributions back to the thread owning that memory page - in this case to thread 0 . When this barrier is complete thread 0 then combines the partial contributions and writes the result to another location in the same page. The second barrier is used to indicate that this operation is complete and that the child processes can now access the final result. As with the basic SCASH synchronization mechanism, the overall cost of a reduction operation will scale as $O$ (No_Threads).

\section{Case Study}

To illustrate the likely performance of SCore cluster enabled OpenMP on a real computational science application code we consider heat distribution in a two dimensional conducting plate. In this problem the temperature of a conducting plate is held constant at the edges and the aim is to determine the temperature of the interior of the plate. The problem is described by the 2-D Laplace equation, and as such is similar to a number of other related problems. The equations are solved iteratively using a finite difference approach with a regular rectangular grid. During each iteration a new value of the temperature at a given grid point is computed based on the average of the temperatures of the four surrounding grid points, with iterations continuing until some agreed convergence is reached. Ignoring convergence testing and imposition of the boundary conditions the basic sequential code is as follows:

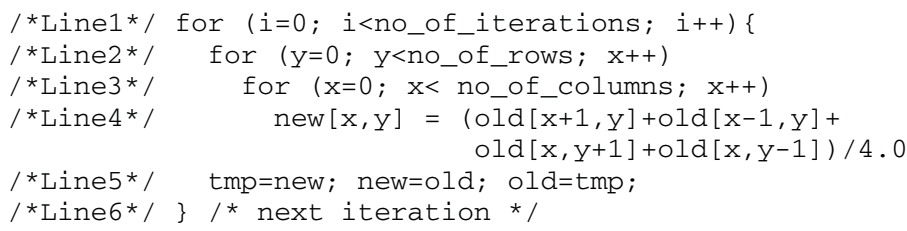

Four parallel implementations were considered:

1. Naïve: A "\#pragma omp parallel for" directive combining thread creation and work division is placed immediately before line2.

2. Barrier minimization: A "\#pragma omp parallel" directive is placed before line 1, and a "\#pragma omp for" before line 2 . The rational for this is that it reduces the number of barriers per iteration from two to one.

3. Page alignment and fault minimization: the memory associated with arrays new and old is carefully allocated so that threads maximize use of "home" data.

4. Barrier and page fault minimzation: optimizations 2 and 3 are combined

Timing results for the four different implementations run on 1, 2 and 4 nodes of the cluster are given in Table 3. From this it is immediately apparent that a naïve inclusion of OpenMP directives into the sequential code is not a good idea. Adjusting the code with the aim of reducing the number of associated barrier calls results in a slight performance gain on 2 nodes, but worse performance on 4 nodes. Since both cases are still much slower than the sequential code there are clearly other factors 
affecting performance. If we now adjust memory to ensure that data quantities are optimally aligned we see a dramatic performance increase, with the code now showing some performance benefit from running on multiple nodes. Finally combining barrier minimization with page placement we obtain the best performance result - albeit only a speedup of 1.9 on 4 nodes of the cluster.

Table 3. Performance comparison of sequential heat code with three alternative OpenMP parallel algorithms run using SCore on the Pentium III cluster for a grid size of 1024x1024 and 100 iterations

\begin{tabular}{lccccc}
\hline & \multicolumn{3}{c}{ Time (sec) } & \multicolumn{2}{c}{ Speedup } \\
Implementation & $\mathbf{1 x 1}$ & $\mathbf{2 x 1}$ & $\mathbf{4 x 1}$ & $\mathbf{2 x 1}$ & $\mathbf{4 x 1}$ \\
\hline 0 Sequential & 8.2 & - & - & & \\
1 Naïve & 8.2 & 75.7 & 86.9 & 0.11 & 0.09 \\
2 Barrier Opt. & 8.2 & 63.7 & 101.1 & 0.13 & 0.08 \\
3 Page Fault Opt. & 8.2 & 6.9 & 5.3 & 1.19 & 1.55 \\
4 Barrier\&Page Opt. & 8.2 & 6.7 & 4.3 & 1.22 & 1.91 \\
\hline
\end{tabular}

\section{Conclusions}

This paper provides a brief overview of the SCore cluster enabled OpenMP environment. The performance of some of the key underlying operations on a cluster of Pentium III processors is evaluated and compared with OpenMP running on a dedicated hardware shared memory system. Using this information and a knowledge of the SCore implementation we were able to obtain an acceptable level of performance for a computational science kernel running on the Pentium III cluster.

Acknowledgements. The authors gratefully acknowledge discussions with J. Antony and A. Over. This work was supported in part by the Australian Research Council through Linkage Grant LP0347178.

\section{References}

1. OpenMP Forum, "OpenMP: A proposed industry standard api for shared memory programming”, http://www.openmp.org, Oct. 1997.

2. Y. Ojima, M. Sato, H. Harada, Y. Ishikawa, "Performance of Cluster-enabled OpenMP for the SCASH Software Distributed Shared Memory System", Proc. of the $3^{\text {rd }}$ IEEE/ACM Int. Sym. on Cluster Computing and the Grid, 450-456 (2003).

3. Y.C. Hu, H. Lu, A.L. Cox, and W. Zwaenepoel, "OpenMP for Networks of SMPs", J. Parallel Dist. Computing, 60, 1512-1530 (2000).

4. S-J. Min, A. Basumallik and R. Eigenmann, "Optimizing OpenMP Programs on Software Distributed Shared Memory Systems”, Int. J. Parallel Programming, 31, 225-249 (2003).

5. D. Margery, G. Vallée, R. Lottiaux, C. Morin, and J-Y. Berthou, "Kerrighed: a SSI Cluster OS Running OpenMP”. Proc. 5th European Workshop on OpenMP (EWOMP '03), Sept. 2003. 
6. L. Huang, B. Chapman, Z. Liu and R. Kendall, "Efficient Translation of OpenMP to Distributed Memory", Lecture Notes in Computer Science, 3038, 408 (2004).

7. See http://www.pccluster.org/

8. L. McVoy, "LMBench - Tools for Performance Analysis", http://www.bitmover.com/ lmbench

9. See http://www.epcc.ed.ac.uk/research/openmpbench/openmp_index.html 